Partial and quasi dynamical symmetries in quantum many-body systems

This content has been downloaded from IOPscience. Please scroll down to see the full text. 2015 J. Phys.: Conf. Ser. 597012003

(http://iopscience.iop.org/1742-6596/597/1/012003)

View the table of contents for this issue, or go to the journal homepage for more

Download details:

IP Address: 132.64.80.234

This content was downloaded on 14/04/2015 at 10:34

Please note that terms and conditions apply. 


\title{
Partial and quasi dynamical symmetries in quantum many-body systems
}

\author{
A Leviatan \\ Racah Institute of Physics, The Hebrew University, Jerusalem 91904, Israel \\ E-mail: ami@phys.huji.ac.il
}

\begin{abstract}
We introduce the notions of partial dynamical symmetry (PDS) and quasi dynamical symmetry (QDS) and demonstrate their relevance to nuclear spectroscopy, to quantum phase transitions and to mixed systems with regularity and chaos. The analysis serves to highlight the potential role of PDS and QDS towards understanding the emergent "simplicity out of complexity" exhibited by complex many-body systems.
\end{abstract}

\section{Introduction}

The concept of dynamical symmetry (DS) is now widely recognized to be of central importance in our understanding of complex many-body systems. It had major impact on developments in diverse areas of physics, including, hadrons, nuclei and molecules [1-3]. Its basic paradigm is to write the Hamiltonian of the system under consideration in terms of Casimir operators of a chain of nested algebras, $G_{0} \supset G_{1} \supset \ldots \supset G_{n}$. The following properties are then observed. (i) All states are solvable and analytic expressions are available for energies and other observables. (ii) All states are classified by quantum numbers, $\left|\alpha_{0}, \alpha_{1}, \ldots, \alpha_{n}\right\rangle$, which are the labels of the irreducible representations (irreps) of the algebras in the chain. (iii) The structure of wave functions is completely dictated by symmetry and is independent of the Hamiltonian's parameters.

The merits of a DS are self-evident. However, in most applications to realistic systems, the predictions of an exact DS are rarely fulfilled and one is compelled to break it. More often one finds that, in a given system, the assumed symmetry is not obeyed uniformly, i.e., is fulfilled by only some states but not by others. In describing a transition between different structural phases, the relevant Hamiltonian, in general, involves competing interactions with incompatible symmetries. The need to address such situations has led to the introduction of partial dynamical symmetry (PDS) [4-6] and quasi dynamical symmetry (QDS) [7-10]. These intermediate-symmetry notions and their implications for dynamical systems, are the subject matter of the present contribution.

\subsection{The interacting boson model}

In order to illustrate the various notions of symmetries and demonstrate their relevance, we employ the interacting boson model (IBM) [2], widely used in the description of low-lying quadrupole collective states in nuclei in terms of $N$ monopole $(s)$ and quadrupole $(d)$ bosons representing valence nucleon pairs. The bilinear combinations $\left\{s^{\dagger} s, s^{\dagger} d_{m}, d_{m}^{\dagger} s, d_{m}^{\dagger} d_{m^{\prime}}\right\}$ span 
a U(6) algebra, which serves as the spectrum generating algebra. The IBM Hamiltonian is expanded in terms of these generators and consists of Hermitian, rotational-scalar interactions which conserve the total number of $s$ - and $d$ - bosons, $\hat{N}=\hat{n}_{s}+\hat{n}_{d}=s^{\dagger} s+\sum_{m} d_{m}^{\dagger} d_{m}$. The three dynamical symmetries of the IBM are

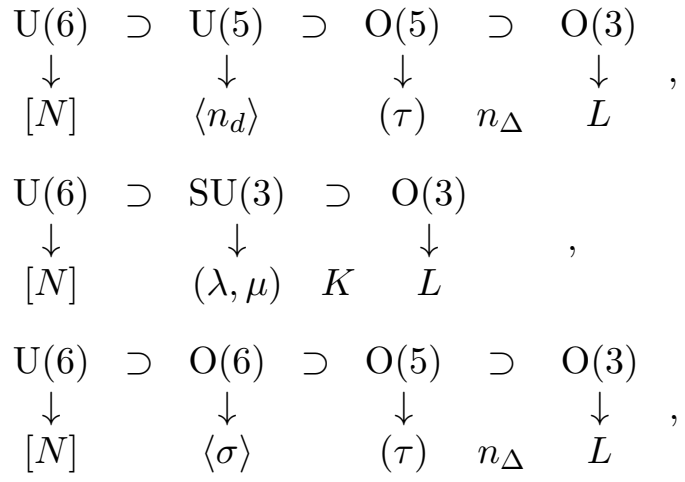

where, below each algebra, its associated labels of irreps are given. $n_{\Delta}$ and $K$ are multiplicity labels needed in the $\mathrm{O}(5) \supset \mathrm{O}(3)$ and $\mathrm{SU}(3) \supset \mathrm{O}(3)$ reductions, respectively. These solvable limits correspond to known benchmarks of the geometric description of nuclei [11], involving vibrational $[\mathrm{U}(5)]$, rotational $[\mathrm{SU}(3)]$ and $\gamma$-soft $[\mathrm{O}(6)]$ types of dynamics.

A geometric visualization of the model is obtained by an energy surface

$$
E_{N}(\beta, \gamma)=\langle\beta, \gamma ; N|\hat{H}| \beta, \gamma ; N\rangle
$$

defined by the expectation value of the Hamiltonian in the coherent (intrinsic) state $[12,13]$

$$
\begin{aligned}
|\beta, \gamma ; N\rangle & =(N !)^{-1 / 2}\left(b_{c}^{\dagger}\right)^{N}|0\rangle, \\
b_{c}^{\dagger} & =\left(1+\beta^{2}\right)^{-1 / 2}\left[\beta \cos \gamma d_{0}^{\dagger}+\beta \sin \gamma\left(d_{2}^{\dagger}+d_{-2}^{\dagger}\right) / \sqrt{2}+s^{\dagger}\right] .
\end{aligned}
$$

Here $(\beta, \gamma)$ are quadrupole shape parameters whose values, $\left(\beta_{\mathrm{eq}}, \gamma_{\mathrm{eq}}\right)$, at the global minimum of $E_{N}(\beta, \gamma)$ define the equilibrium shape for a given Hamiltonian. The shape can be spherical $(\beta=0)$ or deformed $(\beta>0)$ with $\gamma=0$ (prolate), $\gamma=\pi / 3$ (oblate), $0<\gamma<\pi / 3$ (triaxial), or $\gamma$-independent. The equilibrium deformations associated with the dynamical symmetry limits are $\beta_{\mathrm{eq}}=0$ for $\mathrm{U}(5),\left(\beta_{\mathrm{eq}}=\sqrt{2}, \gamma_{\mathrm{eq}}=0\right)$ for $\mathrm{SU}(3)$ and $\left(\beta_{\mathrm{eq}}=1, \gamma_{\mathrm{eq}}\right.$ arbitrary) for $\mathrm{O}(6)$.

One particularly successful approach within the IBM is the extended consistent- $Q$ formalism (ECQF) [14,15], which uses the following Hamiltonian

$$
\hat{H}_{\mathrm{ECQF}}=\omega\left[(1-\xi) \hat{n}_{d}-\frac{\xi}{4 N} \hat{Q}^{\chi} \cdot \hat{Q}^{\chi}\right] .
$$

Here $\hat{Q}^{\chi}=d^{\dagger} s+s^{\dagger} \tilde{d}+\chi\left(d^{\dagger} \tilde{d}\right)^{(2)}$ is the quadrupole operator, $\tilde{d}_{m}=(-)^{m} d_{-m}$ and the dot implies a scalar product. $\xi$ and $\chi$ are the sole structural parameters of the model since $\omega$ is a scaling factor. The parameter ranges $0 \leq \xi \leq 1$ and $-\frac{\sqrt{7}}{2} \leq \chi \leq 0$ interpolate between the $\mathrm{U}(5), \mathrm{O}(6)$ and $\mathrm{SU}(3) \mathrm{DS}$ limits, which are reached for $(\xi, \chi)=(0, \chi),(1,0)$, and $\left(1,-\frac{\sqrt{7}}{2}\right)$, respectively. It is customary to represent the parameter space by a symmetry triangle [16], shown in Fig. 1, whose vertices correspond to these limits. The ECQF has been used extensively for the description of nuclear properties and it was found that the vast majority of nuclei are best described by ECQF parameters in the interior of the triangle, away from any DS limit. In this context, a key question, addressed in the present contribution, can be phrased in the form: are there any remaining "symmetries" inside the triangle? 


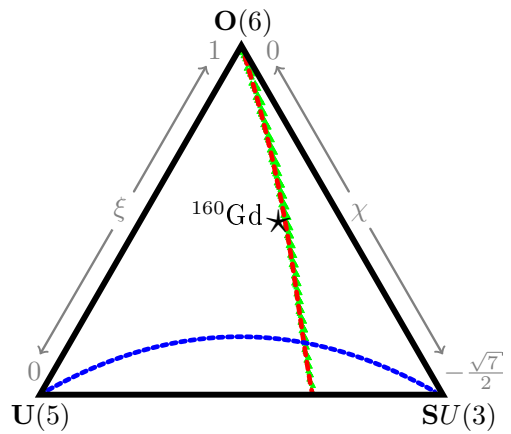

Figure 1. The ECQF symmetry triangle with the position of the nucleus ${ }^{160} \mathrm{Gd}$ indicated by a star. The calculated green curve and its approximation by a red dashed line, correspond to a region of enhanced purity in the ground state, exemplifying an O(6)-PDS discussed in Sections 5.1 and 7. The blue dotted line shows the "arc of regularity" mentioned in Section 8, exemplifying SU(3)-QDS. Adapted from [25]

\section{Partial dynamical symmetry (PDS)}

In algebraic models, such as the IBM, the required symmetry breaking is achieved by including in the Hamiltonian terms associated with (two or more) different sub-algebra chains of the parent spectrum generating algebra. In general, under such circumstances, solvability is lost, there are no remaining non-trivial conserved quantum numbers and all eigenstates are expected to be mixed. A partial dynamical symmetry (PDS) [4-6] corresponds to a particular symmetry breaking for which some (but not all) of the virtues of a dynamical symmetry are retained. The essential idea is to relax the stringent conditions of complete solvability so that the properties (i)-(iii) of a DS, mentioned above, are only partially satisfied. It is then possible to identify the following types of partial dynamical symmetries [6]

- PDS type I:

some of the states have all the dynamical symmetry

- PDS type II:

all the states have part of the dynamical symmetry

- PDS type III:

some of the states have part of the dynamical symmetry.

In PDS of type I, only part of the eigenspectrum is analytically solvable and retains all the dynamical symmetry (DS) quantum numbers. In PDS of type II, the entire eigenspectrum retains some of the DS quantum numbers. PDS of type III has a hybrid character, in the sense that some (solvable) eigenstates keep some of the quantum numbers. In what follows we discuss algorithms for constructing Hamiltonians with partial dynamical symmetries of various types and demonstrate their relevance to quantum many-body systems.

\section{PDS (type I)}

PDS of type I corresponds to a situation for which the defining properties of a dynamical symmetry (DS), namely, solvability, good quantum numbers, and symmetry-dictated structure are fulfilled exactly, but by only a subset of states. An algorithm for constructing Hamiltonians with PDS has been developed in [4] and further elaborated in [5]. The analysis starts from the chain of nested algebras

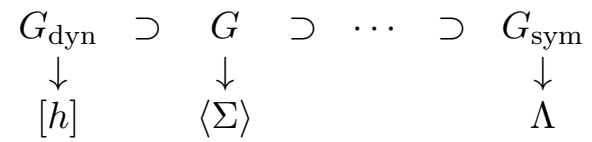

Eq. (5) implies that $G_{\text {dyn }}$ is the dynamical (spectrum generating) algebra of the system such that operators of all physical observables can be written in terms of its generators; a single irrep of $G_{\text {dyn }}$ contains all states of relevance in the problem. In contrast, $G_{\text {sym }}$ is the symmetry algebra and a single of its irreps contains states that are degenerate in energy. Assuming, for simplicity, that particle number is conserved, then all states, and hence the representation $[h]$, can then be assigned a definite particle number $N$. For $N$ identical particles the representation $[h]$ of the dynamical algebra $G_{\text {dyn }}$ is either symmetric $[N]$ (bosons) or antisymmetric $\left[1^{N}\right]$ (fermions) 
and will be denoted, in both cases, as $\left[h_{N}\right]$. The occurrence of a DS of the type (5) signifies that its eigenstates can be labeled as $\left|\left[h_{N}\right]\langle\Sigma\rangle \ldots \Lambda\right\rangle$; additional labels (indicated by ...) are suppressed in the following. Likewise, operators can be classified according to their tensor character under (5) as $\hat{T}_{\left[h_{n}\right]\langle\sigma\rangle \lambda \text {. }}$

Of specific interest in the construction of a PDS associated with the reduction (5), are the $n$-particle annihilation operators $\hat{T}$ which satisfy the property

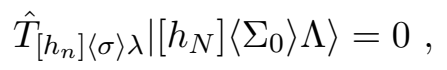

for all possible values of $\Lambda$ contained in a given irrep $\left\langle\Sigma_{0}\right\rangle$ of $G$. Equivalently, this condition can be phrased in terms of the action on a lowest weight (LW) state of the G-irrep $\left\langle\Sigma_{0}\right\rangle$, $\hat{T}_{\left[h_{n}\right]\langle\sigma\rangle \lambda}\left|L W ;\left[h_{N}\right]\left\langle\Sigma_{0}\right\rangle\right\rangle=0$, from which states of good $\Lambda$ can be obtained by projection. Any $n$-body, number-conserving normal-ordered interaction, $\hat{H}=\sum_{\alpha, \beta} A_{\alpha \beta} \hat{T}_{\alpha}^{\dagger} \hat{T}_{\beta}$, written in terms of these annihilation operators and their Hermitian conjugates (which transform as the corresponding conjugate irreps), can be added to the Hamiltonian with a DS (5), while still preserving the solvability of states with $\langle\Sigma\rangle=\left\langle\Sigma_{0}\right\rangle$. If the operators $\hat{T}_{\left[h_{n}\right]\langle\sigma\rangle \lambda}$ span the entire irrep $\langle\sigma\rangle$ of $\mathrm{G}$, then the annihilation condition (6) is satisfied for all $\Lambda$-states in $\left\langle\Sigma_{0}\right\rangle$, if none of the $G$ irreps $\langle\Sigma\rangle$ contained in the $G_{\mathrm{dyn}}$ irrep $\left[h_{N-n}\right]$ belongs to the $G$ Kronecker product $\langle\sigma\rangle \times\left\langle\Sigma_{0}\right\rangle$. So the problem of finding interactions that preserve solvability for part of the states (5) is reduced to carrying out a Kronecker product. The arguments for choosing the special irrep $\langle\Sigma\rangle=\left\langle\Sigma_{0}\right\rangle$ in Eq. (6), which contains the solvable states, are based on physical grounds. A frequently encountered choice is the irrep which contains the ground state of the system.

\section{1. $S U(3) P D S$ (type I) in nuclei}

The SU(3) DS chain of the IBM and related quantum numbers are given in Eq. (1b). The DS Hamiltonian involves the Casimir operators of $\mathrm{SU}(3)$ and $\mathrm{O}(3)$, with eigenvalues $\lambda^{2}+\mu^{2}+\lambda \mu+3(\lambda+\mu)$ and $L(L+1)$, respectively. The spectrum resembles that of an axiallydeformed rotovibrator and the corresponding eigenstates are arranged in $\mathrm{SU}(3)$ multiplets. The label $K$ corresponds geometrically to the projection of the angular momentum on the symmetry axis. In a given $\mathrm{SU}(3)$ irrep $(\lambda, \mu)$, each $K$-value is associated with a rotational band and states with the same angular momentum $L$, in different $K$-bands, are degenerate. The lowest $\mathrm{SU}(3)$ irrep is $(2 N, 0)$, which describes the ground band $g(K=0)$ of a prolate deformed nucleus. The first excited SU(3) irrep $(2 N-4,2)$ contains degenerate $\beta(K=0)$ and $\gamma(K=2)$ bands. This $\beta-\gamma$ degeneracy is a characteristic feature of the $\mathrm{SU}(3)$ limit which, however, is not commonly observed. In most deformed nuclei the $\beta$ band lies above the $\gamma$ band. In the IBM framework, with at most two-body interactions, one is therefore compelled to break $\mathrm{SU}(3)$ in order to conform with the experimental data.

The construction of Hamiltonians with SU(3)-PDS is based on identification of $n$-boson operators which annihilate all states in a given $\mathrm{SU}(3)$ irrep $(\lambda, \mu)$, chosen here to be the ground band irrep $(2 N, 0)$. For that purpose, we consider the following two-boson SU(3) tensors, $B_{[n](\lambda, \mu) K ; L m}^{\dagger}$, with $n=2,(\lambda, \mu)=(0,2)$ and angular momentum $L=0,2$

$$
\begin{aligned}
B_{[2](0,2) 0 ; 00}^{\dagger} & \propto P_{0}^{\dagger}=d^{\dagger} \cdot d^{\dagger}-2\left(s^{\dagger}\right)^{2} \\
B_{[2](0,2) 0 ; 2 m}^{\dagger} & \propto P_{2 m}^{\dagger}=2 d_{m}^{\dagger} s^{\dagger}+\sqrt{7}\left(d^{\dagger} d^{\dagger}\right)_{m}^{(2)} .
\end{aligned}
$$

The corresponding Hermitian conjugate boson-pair annihilation operators, $P_{0}$ and $P_{2 m}$, 
transform as $(2,0)$ under $\mathrm{SU}(3)$, and annihilate all $L$-states in the $(2 N, 0)$ irrep

$$
\begin{aligned}
P_{0}|[N](2 N, 0) K=0, L\rangle & =0, \\
P_{2 m}|[N](2 N, 0) K=0, L\rangle & =0 .
\end{aligned}
$$

Equivalently, these operators annihilate the coherent state, $|\beta=\sqrt{2}, \gamma=0 ; N\rangle$, of Eq. (3), which is the lowest-weight state of this irrep and serves as an intrinsic state for the $\mathrm{SU}(3)$ ground band. The relations in Eq. (8) follow from the fact that the action of the operators $P_{L m}$ leads to a state with $N-2$ bosons in the $\mathrm{U}(6)$ irrep $[N-2]$, which does not contain the $\mathrm{SU}(3)$ irreps obtained from the product $(2,0) \times(2 N, 0)=(2 N+2,0) \oplus(2 N, 1) \oplus(2 N-2,2)$. In addition, $P_{0}$ satisfies

$$
P_{0}|[N](2 N-4 k, 2 k) K=2 k, L M\rangle=0,
$$

where for $k>0$ the indicated $L$-states span only part of the $\mathrm{SU}(3)$ irreps $(\lambda, \mu)=(2 N-4 k, 2 k)$ and form the rotational members of excited $\gamma^{k}(K=2 k)$ bands.

Following the general algorithm, a two-body Hamiltonian with partial SU(3) symmetry can now be constructed as [17]

$$
\hat{H}\left(h_{0}, h_{2}\right)=h_{0} P_{0}^{\dagger} P_{0}+h_{2} P_{2}^{\dagger} \cdot \tilde{P}_{2},
$$

where $\tilde{P}_{2 m}=(-)^{m} P_{2,-m}$. For $h_{2}=h_{0}$, the Hamiltonian is an $\mathrm{SU}(3)$ scalar, related to the quadratic Casimir operator of $\mathrm{SU}(3): h_{2}\left[-\hat{C}_{\mathrm{SU}(3)}+2 \hat{N}(2 \hat{N}+3)\right]$. For $h_{0}=-5 h_{2}$, it transforms as a $(2,2) \mathrm{SU}(3)$ tensor component. Although in general $\hat{H}\left(h_{0}, h_{2}\right)$ is not invariant under $\mathrm{SU}(3)$, Eqs. (8)-(9) ensure that it retains selected solvable states with good SU(3) symmetry, which are members of the ground $g(K=0)$ and $\gamma^{k}(K=2 k)$ bands with the following characteristics

$$
\begin{array}{lll}
|N,(2 N, 0) K=0, L\rangle & E=0 & L=0,2,4, \ldots, 2 N \\
|N,(2 N-4 k, 2 k) K=2 k, L\rangle & E=h_{2} 6 k(2 N-2 k+1) & L=K, K+1, \ldots,(2 N-2 k) .
\end{array}
$$

The remaining eigenstates of $\hat{H}\left(h_{0}, h_{2}\right)$ do not preserve the SU(3) symmetry and therefore get mixed. One can add to $\hat{H}\left(h_{0}, h_{2}\right)$ the Casimir operator of $\mathrm{O}(3), \hat{C}_{\mathrm{O}(3)}$, which contributes an $L(L+1)$ splitting without affecting the wave functions. The resulting Hamiltonian has, by construction, $\mathrm{SU}(3)-\mathrm{PDS}$.

The empirical spectrum of ${ }^{168} \mathrm{Er}$ is shown in Fig. 2 and compared with SU(3)-DS, SU(3)PDS and broken SU(3) calculations [17]. The SU(3)-PDS spectrum shows an improvement over the schematic, exact $\mathrm{SU}(3)$ dynamical symmetry description, since the $\beta-\gamma$ degeneracy is lifted. The quality of the calculated PDS spectrum is similar to that obtained in the broken-SU(3) calculation, however, in the former the ground $g\left(K=0_{1}\right)$ and $\gamma\left(K=2_{1}\right)$ bands remain solvable with good $\mathrm{SU}(3)$ symmetry, $(\lambda, \mu)=(2 N, 0)$ and $(2 N-4,2)$ respectively. At the same time, the excited $K=0_{2}^{+}$band involves about $13 \% \mathrm{SU}(3)$ admixtures into the dominant $(2 N-4,2)$ irrep [18]. Since the wave functions of the solvable states (11) are known, one can obtain analytic expressions for matrix elements of observables between them. The $\mathrm{SU}(3)$ generator, $\hat{Q}$, is obtained from $\hat{Q}^{\chi}$, Eq. (4), for $\chi=-\frac{\sqrt{7}}{2}$, and hence one can write the general E2 operator as $\hat{Q}^{\chi}=\hat{Q}+\theta\left(d^{\dagger} s+s^{\dagger} \tilde{d}\right)$. Since $\hat{Q}$ cannot connect different $\mathrm{SU}(3)$ irreps, only the second term contributes to $\gamma \rightarrow g$ transitions. Accordingly, the calculated B(E2) ratios for these interband transitions are parameter-free predictions of SU(3)-PDS. Based on this observation, an extensive test of SU(3)-PDS was conducted recently [19], showing evidence for its relevance not only for ${ }^{168} \mathrm{Er}$ but also for a wide range of deformed rare earth nuclei. Representative examples of the comparison are shown in Fig. 3. Their detailed analysis provides insights into the complementary role of finite-nucleon number and band-mixing in nuclei. 


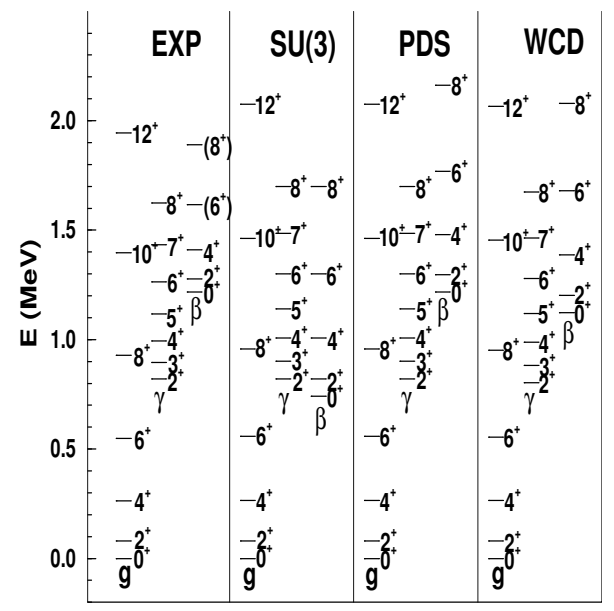

Figure 2. Spectra of ${ }^{168} \operatorname{Er}(N=$ 16). Experimental energies (EXP) are compared with IBM calculations in an exact $\mathrm{SU}(3)$ DS $[\mathrm{SU}(3)]$, in a broken $\mathrm{SU}(3)$ symmetry (WCD) and in SU(3) PDS. The latter employs the Hamiltonian of Eq. (10), $\hat{H}\left(h_{0}, h_{2}\right)+$ $C \hat{C}_{\mathrm{O}(3)}$ with $h_{0}=8, h_{2}=4, C=13$ $\mathrm{keV}$. Adapted from [17].

Figure 3. Comparison of SU(3)-PDS predictions (black bar) with the data (red bar) on the relative $\gamma$-band to ground-band E2 transitions in several rare earth nuclei. Adapted from [19].

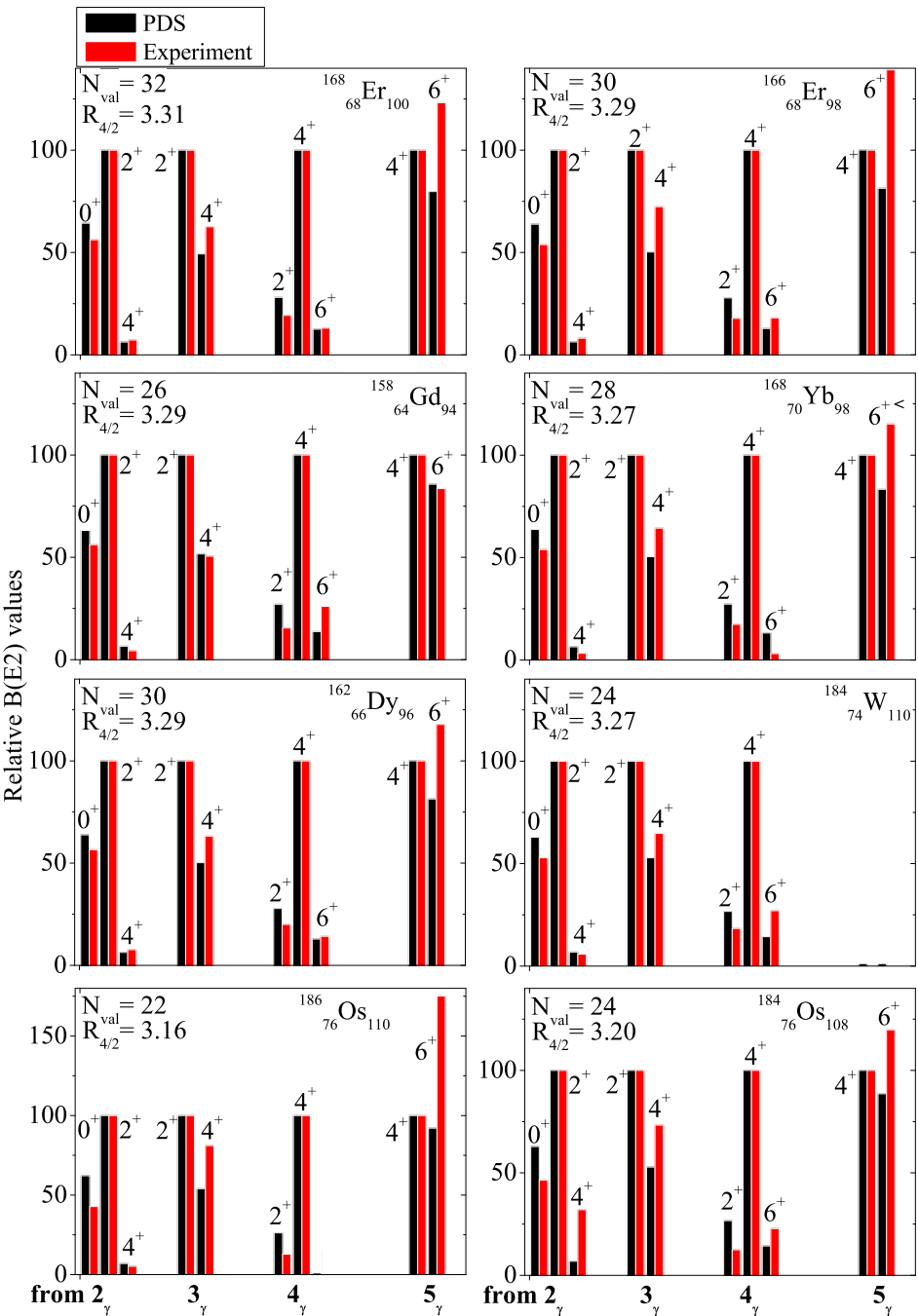

\section{PDS (type II and type III)}

PDS of type II corresponds to a situation for which all the states of the system preserve part of the dynamical symmetry, $G_{0} \supset G_{1} \supset G_{2} \supset \ldots \supset G_{n}$. In this case, there are no analytic solutions, yet selected quantum numbers (of the conserved symmetries) are retained. This occurs, for example, when the Hamiltonian contains interaction terms from two different chains with a common symmetry subalgebra, e.g.,

$$
G_{0} \supset\left\{\begin{array}{l}
G_{1} \\
G_{1}^{\prime}
\end{array}\right\} \supset G_{2} \supset \ldots \supset G_{n} .
$$

If $G_{1}$ and $G_{1}^{\prime}$ are incompatible, i.e., do not commute, then their irreps are mixed in the eigenstates of the Hamiltonian. On the other hand, since $G_{2}$ and its subalgebras are common to both chains, then the labels of their irreps remain as good quantum numbers.

In the IBM, such a situation arises in Hamiltonians combining terms from both the U(5) and $\mathrm{O}(6)$ chains, Eqs. (1a) and (1c) [20]. All eigenstates now mix U(5) irreps $\left(n_{d}\right)$ and $\mathrm{O}(6)$ irreps $\langle\sigma\rangle$, but retain the $(\tau, L)$ labels of the $\mathrm{O}(5) \supset \mathrm{O}(3)$ segment, common to both chains, as good quantum numbers. Hamiltonians of this type have been used in the study of shape-phase transitions between spherical $[\mathrm{U}(5)]$ and $\gamma$-soft $[\mathrm{O}(6)]$ nuclei $[21]$. 
An alternative situation where PDS of type II occurs is when the Hamiltonian preserves only some of the symmetries $G_{i}$ in the DS chain and only their irreps are unmixed. Let $G_{1} \supset G_{2} \supset G_{3}$ be a set of nested algebras which may occur anywhere in the chain, in-between the spectrum generating algebra $G_{0}$ and the invariant symmetry algebra $G_{n}$. A systematic procedure [22] for identifying interactions with PDS of type II, is based on writing the Hamiltonian in terms of generators, $g_{i}$, of $G_{1}$, which do not belong to its subalgebra $G_{2}$. By construction, such Hamiltonian preserves the $G_{1}$ symmetry but, in general, not the $G_{2}$ symmetry, and hence will have the $G_{1}$ labels as good quantum numbers but will mix different irreps of $G_{2}$. The Hamiltonians can still conserve the $G_{3}$ labels e.g., by choosing it to be a scalar of $G_{3}$. The procedure involves the identification of the tensor character under $G_{2}$ and $G_{3}$ of the operators $g_{i}$ and their products, $g_{i} g_{j} \ldots g_{k}$. The Hamiltonians obtained in this manner belong to the integrity basis of $G_{3}$-scalar operators in the enveloping algebra of $G_{1}$ and, hence, their existence is correlated with their order.

In the IBM, such a scenario can be realized by considering an interaction term of the form $\left(\left(\Pi^{(2)} \times \Pi^{(2)}\right)^{(2)} \cdot \Pi^{(2)}\right.$, constructed from the $\mathrm{O}(6)$ generator, $\Pi^{(2)}=d^{\dagger} s+s^{\dagger} \tilde{d}$, which is not a generator of $\mathrm{O}(5)$ [22]. Such a term cannot connect states in different $\mathrm{O}(6)$ irreps but can induce $\mathrm{O}(5)$ mixing subject to $\Delta \tau= \pm 1, \pm 3$. Consequently, all eigenstates of the resulting Hamiltonian have good $\mathrm{O}(6)$ quantum number $\sigma$ but do not possess $\mathrm{O}(5)$ symmetry $\tau$. These are the necessary ingredients of an O(6) PDS of type II associated with the chain of Eq. (1c).

PDS of type III has a hybrid character, for which some of the states of the system under study preserve part of the dynamical symmetry [23]. In relation to the dynamical symmetry chain of Eq. (5), with associated basis, $\left|\left[h_{N}\right]\langle\Sigma\rangle \Lambda\right\rangle$, this can be accomplished by relaxing the condition of Eq. (6), so that it holds only for selected states $\Lambda$ contained in a given irrep $\left\langle\Sigma_{0}\right\rangle$ of $G$ and/or selected (combinations of) components $\lambda$ of the tensor $\hat{T}_{\left[h_{n}\right]\langle\sigma\rangle \lambda}$. Under such circumstances, let $G^{\prime} \neq G_{\text {sym }}$ be a subalgebra of $G$ in the aforementioned chain, $G \supset G^{\prime}$. In general, the Hamiltonians, constructed from these tensors, in the manner described in Section 3, are not invariant under $G$ nor $G^{\prime}$. Nevertheless, they do possess the subset of solvable states, $\left|\left[h_{N}\right]\left\langle\Sigma_{0}\right\rangle \Lambda\right\rangle$, with good $G$-symmetry $\left\langle\Sigma_{0}\right\rangle$ (which now span only part of the corresponding $G$ irrep), while other states are mixed. At the same time, the symmetry associated with the subalgebra $G^{\prime}$, is broken in all states (including the solvable ones). Thus, part of the eigenstates preserve part of the symmetry. These are precisely the requirements of PDS of type III.

In the IBM, such a generalized partial symmetry associated with the $\mathrm{O}(6)$ chain of Eq. (1c), can be realized by an Hamiltonian constructed of boson-pair operators which are not invariant under $\mathrm{O}(6)$ nor $\mathrm{O}(5)$, but annihilate the coherent state, $|\beta=1, \gamma=0 ; N\rangle$, of Eq. (3), which has $\sigma=N$ [23]. Such an Hamiltonian has a solvable ground band with good $\mathrm{O}(6)$ symmetry, which is not preserved by other states. All eigenstates, including the solvable ones, break the $\mathrm{O}(5)$ symmetry. An empirical manifestation of such type of O(6)-PDS is presented in Section 5.1.

\section{Measures of PDS}

The PDS notion reflects the purity of selected eigenstates with respect to a DS basis. The above algorithms provide a procedure for an explicit construction of Hamiltonians with such property. More general (and realistic) Hamiltonians often exhibit features of a PDS to a certain approximation. In such cases, one needs to assess the quality and applicability of the PDS notion. In what follows, we discuss two quantitative measures of PDS, based on wave-function entropy and quantum number fluctuations.

Consider an eigenfunction of the IBM Hamiltonian, $|L\rangle$, with angular momentum $L$. Its expansion in a DS basis reads $|L\rangle=\sum_{i} C_{i}\left|[N], \alpha_{i}, L\right\rangle$, where $C_{i}$ stands for the expansion coefficients $C_{n_{d}, \tau, n_{\Delta}}^{(L)}, C_{(\lambda, \mu), K}^{(L)}, C_{\sigma, \tau, n_{\Delta}}^{(L)}$, in the $\mathrm{U}(5), \mathrm{SU}(3), \mathrm{O}(6)$ bases, Eq. (1), respectively. 
The probability distributions of $\mathrm{U}(5): P_{n_{d}}^{(L)}, \mathrm{SU}(3): P_{(\lambda, \mu)}^{(L)}$, and $\mathrm{O}(6): P_{\sigma}^{(L)}$, are calculated as

$$
\begin{array}{ll}
P_{n_{d}}^{(L)}=\sum_{\tau, n_{\Delta}}\left|C_{n_{d}, \tau, n_{\Delta}}^{(L)}\right|^{2} \quad, & S_{\mathrm{U} 5}(L)=-\sum_{n_{d}} P_{n_{d}}^{(L)} \ln P_{n_{d}}^{(L)}, \\
P_{(\lambda, \mu)}^{(L)}=\sum_{K}\left|C_{(\lambda, \mu), K}^{(L)}\right|^{2} \quad, \quad S_{\mathrm{SU} 3}(L)=-\sum_{(\lambda, \mu)} P_{(\lambda, \mu)}^{(L)} \ln P_{(\lambda, \mu)}^{(L)}, \\
P_{\sigma}^{(L)}=\sum_{\tau, n_{\Delta}}\left|C_{\sigma, \tau, n_{\Delta}}^{(L)}\right|^{2} \quad, \quad S_{\mathrm{O} 6}(L)=-\sum_{\sigma} P_{\sigma}^{(L)} \ln P_{\sigma}^{(L)} .
\end{array}
$$

The indicated Shannon state entropy $S_{G}(L),[G=\mathrm{U}(5), \mathrm{SU}(3), \mathrm{O}(6)]$ is a convenient tool to evaluate the purity of eigenstates with respect to a DS basis [24]. It vanishes when the considered state is pure with good $G$-symmetry $\left[S_{\mathrm{G}}(L)=0\right]$, and is positive for a mixed state. Normalized entropies are obtained by dividing by $\ln D_{G}$, where $D_{G}$ counts the number of possible $G$-irreps for a given $L$. In this case, the maximal value $\left[S_{\mathrm{G}}(L)=1\right]$ is obtained when $|L\rangle$ is uniformly spread among the irreps of $G$, i.e., for $P_{G}^{(L)}=1 / D_{G}$. Intermediate values, $0<S_{\mathrm{G}}(L)<1$, indicate partial fragmentation of the state $|L\rangle$ in the respective DS basis.

The degree of a symmetry of a state $|L\rangle$ can also be inferred from the fluctuations of the corresponding quantum number. As an example, for the $\mathrm{O}(6)$ symmetry of the IBM, the fluctuations in $\sigma$ can be calculated as [25]

$$
\Delta \sigma_{L}=\sqrt{\sum_{i} C_{i}^{2} \sigma_{i}^{2}-\left(\sum_{i} C_{i}^{2} \sigma_{i}\right)^{2}},
$$

where the sum is over all basis states in the chain, Eq. (1c). If $|L\rangle$ carries an exact $\mathrm{O}(6)$ quantum number, $\sigma$ fluctuations are zero, $\Delta \sigma_{L}=0$. If $|L\rangle$ contains basis states with different $\mathrm{O}(6)$ quantum numbers, then $\Delta \sigma_{L}>0$, indicating that the $\mathrm{O}(6)$ symmetry is broken. Note that $\Delta \sigma_{L}$ also vanishes for a state with a mixture of components with the same $\sigma$ but different $\mathrm{O}(5)$ quantum numbers $\tau$, corresponding to a $|L\rangle$ with good $\mathrm{O}(6)$ but mixed $\mathrm{O}(5)$ character. $\Delta \sigma_{L}$ has the same physical content as $S_{O 6}(L)(13 \mathrm{c})$ and both can be used as measures of O(6)-PDS.

\section{1. $O(6)-P D S$ (type III) in nuclei}

A recent study [25] has examined the fluctuations $\Delta \sigma_{L}$, Eq. (14), for the entire parameter space of the ECQF Hamiltonian (4). Results of this calculation for the ground state, $\left|L=0_{1}^{+}\right\rangle$, with $N=14$, are shown in Fig. 4. At the O(6) DS limit $(\xi=1, \chi=0), \Delta \sigma_{0} \equiv \Delta \sigma_{L=0_{1}}$ vanishes per construction whereas it is greater than zero for all other parameter pairs. Towards the $\mathrm{U}(5)$ DS limit $(\xi=0)$, the fluctuations reach a saturation value of $\Delta \sigma_{0} \approx 2.47$. At the SU(3) DS limit $\left(\xi=1, \chi=-\frac{\sqrt{7}}{2}\right)$ the fluctuations are $\Delta \sigma_{0} \approx 1.25$. Surprisingly, one recognizes a valley of almost vanishing $\Delta \sigma_{0}$ values, two orders of magnitude lower than at saturation. This region (depicted by a green arc in the triangle of Fig. 1), represents a parameter range of the IBM, outside the $\mathrm{O}(6)$ DS limit, where the ground-state wave function exhibits an exceptionally high degree of purity with respect to the $\mathrm{O}(6)$ quantum number $\sigma$.

The ground-state wave functions in the valley of low $\Delta \sigma_{0}$, can be expanded in the O(6)-DS basis (1c). At the $\mathrm{O}(6)$ DS limit only one $\mathrm{O}(6)$ basis state, with $\sigma=N$ and $\tau=0$ contributes, while outside this limit the wave function consists of multiple $\mathrm{O}(6)$ basis states. Investigation of the wave function for parameter combinations inside the valley reveals an overwhelming dominance of the $\mathrm{O}(6)$ basis states with $\sigma=N$. This is seen in Fig. 5 for the ground-state wave function of $\hat{H}_{\mathrm{ECQF}}(4)$, with parameter values that apply to the nucleus ${ }^{160} \mathrm{Gd}$. The $\sigma=N$ 


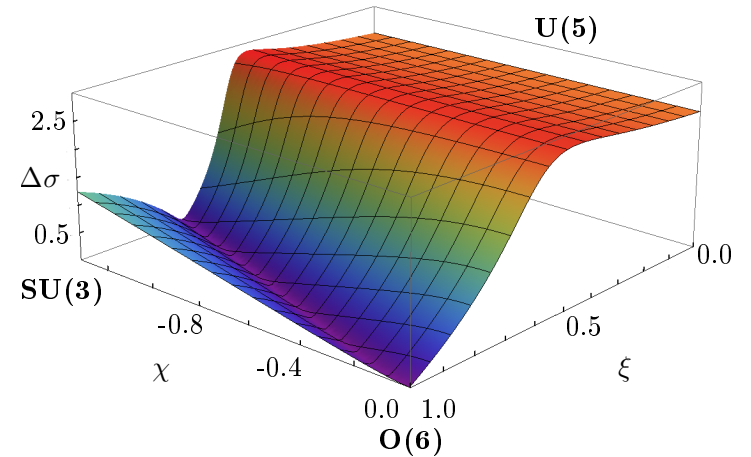

Figure 4. Ground-state $\left(L=0_{1}^{+}\right)$fluctuations $\Delta \sigma_{0}(14)$ for $\hat{H}_{\mathrm{ECQF}}(4)$ with $N=14$. The fluctuations vanish at the $\mathrm{O}(6)$ DS limit, saturate towards the U(5) DS limit, and are of the order $10^{-2}$ in the valley. Adapted from [25].

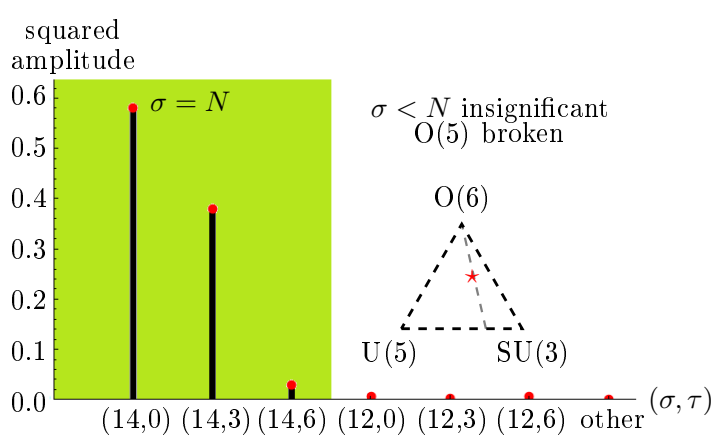

Figure 5. Squared amplitudes $C_{i}^{2}$ in the expansion in the $\mathrm{O}(6)$ basis (1c), of the $L=0_{1}^{+}$ ground state of the ECQF Hamiltonian (4) with parameters $\xi=0.84, \chi=-0.53, N=14$, appropriate for ${ }^{160} \mathrm{Gd}$. Adapted from [25].

Table 1. Calculated $\sigma$ fluctuations $\Delta \sigma_{L}$, Eq. (14), for rare earth nuclei in the vicinity of the identified region of approximate ground-state- $\mathrm{O}(6)$ symmetry [25]. Also shown are the fraction $f_{\sigma=\mathrm{N}}^{(L)}$ of $\mathrm{O}(6)$ basis states with $\sigma=N$ contained in the $L=0,2,4$ states, members of the ground band. The structure parameters $\xi$ and $\chi$ are taken from $[26]$.

\begin{tabular}{lccccccccc}
\hline Nucleus & $N$ & $\xi$ & $\chi$ & $\Delta \sigma_{0}$ & $f_{\sigma=\mathrm{N}}^{(0)}$ & $\Delta \sigma_{2}$ & $f_{\sigma=\mathrm{N}}^{(2)}$ & $\Delta \sigma_{4}$ & $f_{\sigma=\mathrm{N}}^{(4)}$ \\
\hline${ }^{156} \mathrm{Gd}$ & 12 & 0.72 & -0.86 & 0.46 & $95.3 \%$ & 0.43 & $95.8 \%$ & 0.38 & $96.6 \%$ \\
${ }^{158} \mathrm{Gd}$ & 13 & 0.75 & -0.80 & 0.35 & $97.2 \%$ & 0.33 & $97.5 \%$ & 0.30 & $97.9 \%$ \\
${ }^{160} \mathrm{Gd}$ & 14 & 0.84 & -0.53 & 0.19 & $99.1 \%$ & 0.19 & $99.2 \%$ & 0.17 & $99.3 \%$ \\
${ }^{162} \mathrm{Gd}$ & 15 & 0.98 & -0.53 & 0.41 & $96.0 \%$ & 0.40 & $96.0 \%$ & 0.40 & $96.1 \%$ \\
${ }^{160} \mathrm{Dy}$ & 14 & 0.81 & -0.49 & 0.44 & $96.2 \%$ & 0.39 & $96.4 \%$ & 0.36 & $96.8 \%$ \\
${ }^{162} \mathrm{Dy}$ & 15 & 0.92 & -0.31 & 0.07 & $99.9 \%$ & 0.07 & $99.9 \%$ & 0.06 & $99.9 \%$ \\
${ }^{164} \mathrm{Dy}$ & 16 & 0.98 & -0.26 & 0.13 & $99.6 \%$ & 0.13 & $99.6 \%$ & 0.13 & $99.6 \%$ \\
${ }^{164} \mathrm{Er}$ & 14 & 0.84 & -0.37 & 0.39 & $96.5 \%$ & 0.37 & $96.7 \%$ & 0.35 & $97.1 \%$ \\
${ }^{166} \mathrm{Er}$ & 15 & 0.91 & -0.31 & 0.12 & $99.7 \%$ & 0.11 & $99.7 \%$ & 0.10 & $99.7 \%$ \\
\hline
\end{tabular}

states comprise more than $99 \%$ of the ground-state wave function at the bottom of the valley and their dominance causes $\Delta \sigma_{0}$ to be small. At the same time, the $\mathrm{O}(5)$ symmetry is broken, as basis states with different quantum number $\tau$ contribute significantly to the wave function. Consequently, the valley can be identified as an entire region in the symmetry triangle with an approximate $\mathrm{O}(6)$-PDS of type III. Outside this valley the ground state is a mixture of several $\sigma$ values and $\Delta \sigma_{0}$ increases.

Detailed ECQF fits for energies and electromagnetic transitions of rare-earth nuclei, performed in [26], allow one to relate the structure of collective nuclei to the parameter space of the ECQF Hamiltonian (4). From the extracted $(\xi, \chi)$ parameters one can calculate the fluctuations $\Delta \sigma_{L}$ and the fractions $f_{\sigma=N}$ of squared $\sigma=N$ amplitude. Nuclei with $\Delta \sigma_{0}<0.5$ and $f_{\sigma=N}>95 \%$ in the ground-state $\left(L=0_{1}^{+}\right)$are listed in Table 1. These quantities are also calculated for yrast states with $L>0$ and exhibit similar values in each nucleus. It is evident that a large set of rotational rare earth nuclei, such as ${ }^{160} \mathrm{Gd}$, are located in the valley of small $\sigma$ fluctuations. They can be identified as candidate nuclei with an approximate O(6)-PDS of type III not only for the ground state, but also for the members of the band built on top of it. 


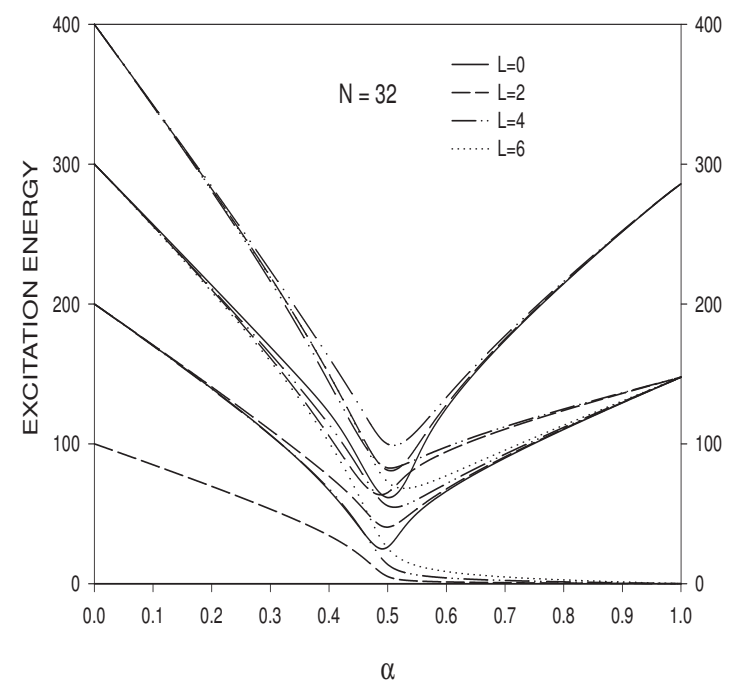

Figure 6. Spectrum of $\hat{H}(\alpha)$, Eq. (15), interpolating between the $\mathrm{U}(5)(\alpha=0)$ and $\mathrm{SU}(3)(\alpha=1)$ DS limits. From [29].

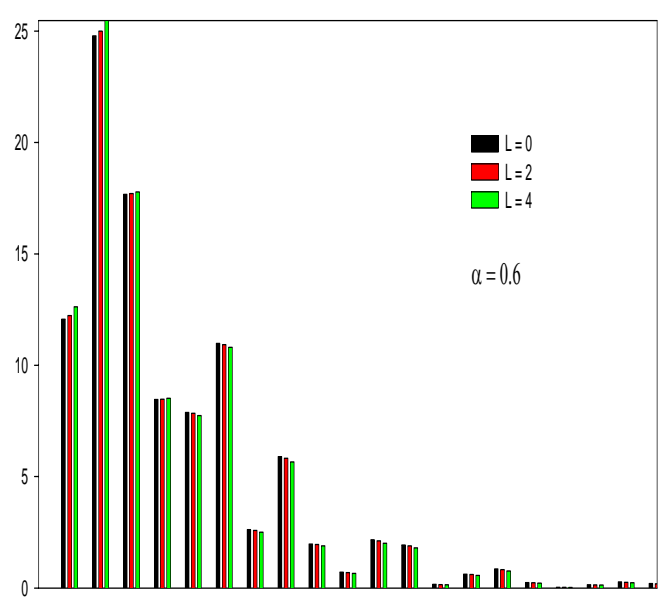

Figure 7. Squared amplitudes for angular momenta $L=0,2,4$ yrast eigenstates of the $\mathrm{U}(5)$ $\mathrm{SU}(3)$ Hamiltonian $\hat{H}(\alpha)$, Eq. (15), with $\alpha=0.6$, in the $\mathrm{SU}(3)$ basis (1b). From [29]

\section{Quasi dynamical symmetry (QDS)}

A second kind of intermediate-symmetry occurring in algebraic modeling of dynamical systems, is that of quasi dynamical symmetry (QDS) [7-10]. While QDS can be defined mathematically in terms of embedded representations $[27,28]$, its physical meaning is that several observables associated with particular eigenstates, may be consistent with a certain symmetry which in fact is broken in the Hamiltonian. This typically occurs for a Hamiltonian transitional between two DS limits

$$
\hat{H}(\alpha)=(1-\alpha) \hat{H}_{\mathrm{G}_{1}}+\alpha \hat{H}_{\mathrm{G}_{2}} .
$$

$\hat{H}(\alpha)$ involves competing incompatible (non-commuting) symmetries. For $\alpha=0$ or $\alpha=1$, one recovers the limiting symmetries. For $0<\alpha<1$, both symmetries are broken and mixing occurs. A detailed study [8-10] of such Hamiltonians has found that for most values of $\alpha$, selected states continue to exhibit characteristic properties (e.g., energy and $\mathrm{B}(\mathrm{E} 2)$ ratios) of the closest $\mathrm{DS}$ limit. Such an "apparent" persistence of symmetry in the face of strong symmetry-breaking interactions, defines a QDS. The indicated persistence is clearly evident in the spectrum shown in Fig. 6, for an IBM Hamiltonian, $\hat{H}(\alpha)$, interpolating between the $G_{1}=U(5)$ and $G_{2}=S U(3)$ DS limits, relevant to shape-phase transitions between spherical and axially-deformed nuclei [21]. The "apparent" symmetry is due to the coherent nature of the mixing. As seen in Fig. 7, the mixing of SU(3) irreps is large, but is approximately independent of the angular momentum of the yrast states, i.e., the $\mathrm{SU}(3)$ expansion coefficients $C_{(\lambda, \mu), K}^{(L)} \approx$ independent of $L$. The set of states exhibiting SU(3)-QDS thus have a common structure and form a band (the ground band) associated with a single intrinsic state.

The criterion for the validity of QDS is the similarity of the decomposition in the given DS basis. Thus, in the IBM, a quantitative measure of SU(3)-QDS can be defined as $\sqrt{1-\bar{\Theta}}$ [30], where $\bar{\Theta}$ is the average of $\Theta_{L L^{\prime}}=\sum_{i} C_{i}^{L} C_{i}^{L^{\prime}}$ for all pairs $L \neq L^{\prime}$, and $C_{i}^{L}$ are the amplitudes of the chosen set of eigenstates with angular momentum $L$ in the $\mathrm{SU}(3)$ basis (1b).

The coherent decomposition signaling SU(3) QDS, implies strong correlations between the $\mathrm{SU}(3)$ components of different $L$-states in the same band. This can be used as an alternative criterion for the identification of rotational bands with SU(3)-QDS [31]. Focusing on the 

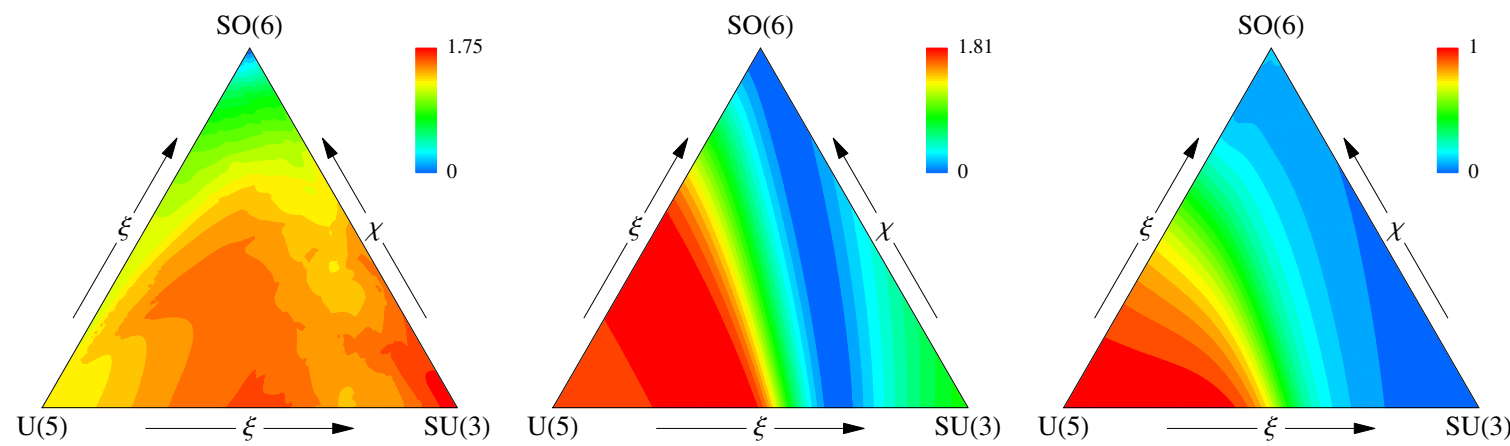

Figure 8. PDS and QDS for eigenstates of the ECQF Hamiltonian, Eq. (4), with $N=15$. Left panel: the $\mathrm{O}(6)$-PDS measure [Shannon entropy $S_{\mathrm{O} 6}(L)$, Eq. (13c)], averaged for all $L=0$ eigenstates. Middle panel: $S_{\mathrm{O} 6}(L)$ for the ground state $L=0_{1}$. Right panel: the quantity $\sqrt{1-\bar{\Theta}}$, which is the SU(3)-QDS measure (discussed in the text) for yrast (lowest energy) states with $L=0,2, \ldots, 10$. From [32].

$L=0,2,4,6$, members of $K=0$ bands, given a $L=0_{i}^{+}$state, among the ensemble of possible states, we associate with it those $L_{j}>0$ states which show the maximum correlation, $\max _{j}\left\{\pi\left(0_{i}, L_{j}\right)\right\}$. Here $\pi\left(0_{i}, L_{j}\right)$ is a Pearson coefficient whose values lie in the range $[-1,1]$. Specifically, $\pi\left(0_{i}, L_{j}\right)=1,-1,0$, indicate a perfect correlation, a perfect anti-correlation, and no linear correlation, respectively, among the $\mathrm{SU}(3)$ components of the $0_{i}$ and $L_{j}$ states. To quantify the amount of coherence (hence of SU(3)-QDS) in the chosen set of states, one can adapt the procedure of [31] and employ the following product of the maximum correlation coefficients: $C_{\mathrm{SU} 3}\left(0_{i}-6\right) \equiv \max _{j}\left\{\pi\left(0_{i}, 2_{j}\right)\right\} \max _{k}\left\{\pi\left(0_{i}, 4_{k}\right)\right\} \max _{\ell}\left\{\pi\left(0_{i}, 6_{\ell}\right)\right\}$. The set of states $\left\{0_{i}, 2_{j}, 4_{k}, 6_{\ell}\right\}$ is considered as comprising a $K=0$ band with $\mathrm{SU}(3)$-QDS, if $C_{\mathrm{SU} 3}\left(0_{i}-6\right) \approx 1$.

\section{1. $P D S$ and $Q D S$ in the symmetry triangle}

Recently, a comprehensive analysis of the PDS and QDS properties of $\hat{H}_{\mathrm{ECQF}}$, Eq. (4), was carried out [30], employing the symmetry measures discussed above. Representative results are shown in Fig. 8. The left panel of Fig. 8, shows the $\mathrm{O}(6)$ wave function entropy, $S_{\mathrm{O} 6}(L=0)$, Eq. (13c), averaged over all $L=0$ eigenstates. As seen, only a small region (marked in blue) near the $\mathrm{O}(6)$ vertex where an exact $\mathrm{O}(6)$ DS occurs, shows a high degree of purity with respect to the $\mathrm{O}(6)$ quantum number, $\sigma$. The middle panel displays $S_{\mathrm{O} 6}\left(L=0_{1}\right)$ of only the lowest $L=0_{1}$ state. Here, $\sigma$ is conserved in the ground state throughout an entire region (marked in blue) of ECQF Hamiltonians, reflecting the O(6)-PDS of type III discussed in Section 5.1. The right panel displays the QDS measure $\sqrt{1-\bar{\Theta}}$, with respect to the $\mathrm{SU}(3)$ basis. It is seen that large areas of the triangle are blue, i.e., display SU(3)-QDS. These results illustrate the wider applicability in nuclei of the extended concepts, PDS and QDS, as compared to an exact DS.

\section{Linking PDS and QDS}

The concept of PDS reflects the purity of selected states, hence is different from the concept of QDS which reflects a coherent mixing. Nevertheless, a link between these two hitherto unrelated symmetry concepts can be established and shown to be empirically manifested in rotational nuclei $[25]$.

The experimental spectrum of ${ }^{160} \mathrm{Gd}$, along with its ECQF description (4), is shown in the left panel of Fig. 9. The middle and right panels show the decomposition into $\mathrm{O}(6)$ and $\mathrm{SU}(3)$ basis states, respectively, for yrast states with $L=0,2,4$. It is evident that the $\mathrm{SU}(3)$ symmetry is broken, as significant contributions of basis states with different $\mathrm{SU}(3)$ quantum numbers $(\lambda, \mu)$ occur. It is also clear from Fig. 9c that this mixing occurs in a coherent manner with similar patterns for the different members of the ground-state band. As explained in Section 6, 


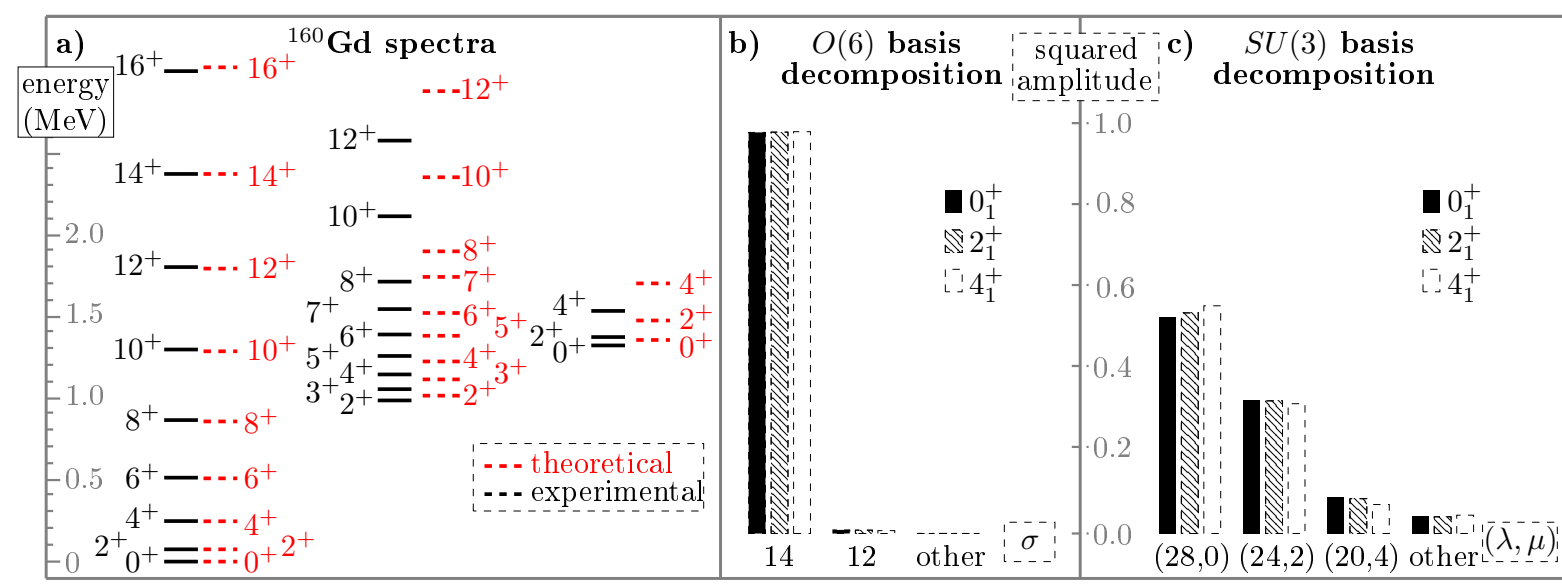

Figure 9. a) The experimental spectrum of ${ }^{160} \mathrm{Gd}$ compared with IBM calculation using $\hat{H}_{\mathrm{ECQF}}$ (4) with $\xi=0.84, \chi=-0.5, N=14$. b) The $\mathrm{O}(6)$ decomposition in $\sigma$ components of yrast states with $L=0,2,4$. c) The $\mathrm{SU}(3)$ decomposition in $(\lambda, \mu)$ components of the same states. Adapted from [25].

this is the hallmark of SU(3) QDS. On the other hand, as seen in Fig. 9b, the yrast states with $L=0,2,4$ are almost entirely composed out of $\mathrm{O}(6)$ basis states with $\sigma=N=14$ which implies small fluctuations $\Delta \sigma_{L}(14)$ and the preservation of $\mathrm{O}(6)$ symmetry in the ground-state band. At the same time, as shown in Fig. 5, the $\mathrm{O}(5)$ symmetry is broken in these states. Thus an empirically-manifested link is established between SU(3) QDS and O(6) PDS of type III.

The SU(3) QDS property for the members of the ground band results from the existence of a single intrinsic state, which is the coherent state, $|\beta=1, \gamma=0 ; N\rangle$ of Eq. (3) that has $\sigma=N$. Indeed, the $(\xi, \chi)$ parameter range of the ECQF Hamiltonian for which the equilibrium deformations are $(\beta=1, \gamma=0)$, shown by a red dashed line in Fig. 1, coincides with the region of an approximate ground-state $\mathrm{O}(6)$ symmetry for large $N$. These results demonstrate that coherent mixing of one symmetry (QDS) can result in the purity of a quantum number associated with partial conservation of a different, incompatible symmetry (PDS).

\section{Impact of PDS and QDS on mixed regular and chaotic dynamics}

Hamiltonians with a dynamical symmetry are always completely integrable. The Casimir invariants of the algebras in the chain provide a set of constants of the motion in involution. The classical motion is purely regular. A symmetry-breaking is connected to non-integrability and may give rise to chaotic motion. Hamiltonians with PDS and QDS are not completely integrable, hence can exhibit stochastic behavior, nor are they completely chaotic, since some eigenstates preserve the symmetry exactly in a PDS or mix in a coherent fashion in a QDS. Consequently, Hamiltonians with such intermediate symmetries are optimally suitable to the study of mixed systems with coexisting regularity and chaos.

The dynamics of a generic classical Hamiltonian system is mixed; KAM islands of regular motion and chaotic regions coexist in phase space. In the associated quantum system, the statistical properties of the spectrum are usually intermediate between the Poisson and the Gaussian orthogonal ensemble (GOE) statistics. In a PDS, the symmetry of the subset of solvable states is exact, yet does not arise from invariance properties of the Hamiltonian. Several works have shown that a PDS is strongly correlated with suppression of chaos $[33,34]$. This enhancement of regularity was seen in both the classical measures of chaos, e.g., the fraction of chaotic volume and the average largest Lyapunov exponent, and in quantum measures of chaos, e.g., the nearest neighbors level spacing distribution, whose parameter interpolates between the 
Poisson and GOE statistics. The reduction in chaos occurs even when the fraction of solvable states approaches zero in the classical limit, suggesting that the existence of a PDS increases the purity of other neighbouring states in the system.

The coherent mixing common to a set of states, characterizing a QDS, results from the existence of a single intrinsic state for each such band and imprints an adiabatic motion and increased regularity [35]. This was verified for low- [10] and high-lying [31] rotational bands using the ECQF Hamiltonian, Eq. (4). SU(3) QDS has been proposed [36] to underly the "arc of regularity" [37], a narrow zone of enhanced regularity in the parameter-space of $\hat{H}_{\mathrm{ECQF}}$. The arc, shown by a blue dotted line in Fig. 1, resides in the interior of the symmetry triangle and connects the $\mathrm{U}(5)$ and $\mathrm{SU}(3)$ vertices.

\section{PDS and QDS in a first-order quantum phase transition}

Quantum phase transitions (QPTs) are qualitative changes in the properties of a physical system induced by a variation of parameters in the quantum Hamiltonian. Such structural changes are currently of great interest in different branches of physics [38]. The competing interactions in the Hamiltonian that drive these ground-state phase transitions can affect dramatically the nature of the dynamics and, in some cases, lead to the emergence of quantum chaos [39-42]. Here we show that PDS and QDS can characterize the remaining regularity in a system undergoing a QPT, amidst a complicated environment of other states [42].

Focusing on the dynamics at the critical-point of a first-order QPT between spherical and deformed shapes, the relevant IBM Hamiltonian [43], upto a scale, can be taken to be the second term of Eq. (10), $\hat{H}_{\text {cri }}=P_{2}^{\dagger} \cdot \tilde{P}_{2}$. The latter has the SU(3) basis states of Eq. (11) and the following $\mathrm{U}(5)$ basis states

$$
\begin{array}{ll}
\left|N, n_{d}=\tau=L=0\right\rangle & E=0, \\
\left|N, n_{d}=\tau=L=3\right\rangle & E=6(2 N-1),
\end{array}
$$

as solvable eigenstates, while all other states are mixed with respect to both $\mathrm{U}(5)$ and $\mathrm{SU}(3)$. As such, $\hat{H}_{\text {cri }}$ exhibits a coexistence of SU(3)-PDS and U(5)-PDS [44].

The classical limit of the Hamiltonian is obtained through the use of Glauber coherent states. This amounts to replacing $\left(s^{\dagger}, d_{\mu}^{\dagger}\right)$ by c-numbers $\left(\alpha_{s}^{*}, \alpha_{\mu}^{*}\right)$ rescaled by $\sqrt{N}$ and taking $N \rightarrow \infty$, with $1 / N$ playing the role of $\hbar$ [45]. Setting all momenta to zero, yields the classical potential $V(x, y)$, which coincides with the surface of Eq. $(2)$, with $(\beta, \gamma)$ as polar coordinates. The classical dynamics constraint to $L=0$, can be depicted conveniently via Poincare surfaces of sections in the plane $y=0$, plotting the values of $\left(p_{x}, x\right)$ each time a trajectory intersects the plane. Regular trajectories are bound to toroidal manifolds within the phase space and their intersections with the plane of section lie on $1 \mathrm{D}$ curves (ovals). In contrast, chaotic trajectories randomly cover kinematically accessible areas of the section.

The Poincaré sections associated with the classical critical-point Hamiltonian are shown in Fig. 10 for representative energies. The bottom panel displays the classical potential which has two degenerate spherical and deformed minima. The dynamics in the region of the deformed minimum is robustly regular. The trajectories form a single island and remain regular even at energies far exceeding the barrier height $V_{\mathrm{bar}}$. In contrast, the dynamics in the region of the spherical minimum shows a change with energy from regularity to chaos, until complete chaoticity is reached near the barrier top. The clear separation between regular and chaotic dynamics, associated with the two minima, persists all the way to the barrier energy, $E=V_{\text {bar }}$, where the two regions just touch. At $E>V_{\mathrm{bar}}$, a layer of chaos develops in the deformed region and gradually dominates the surviving regular island for $E \gg V_{b}$.

The quantum manifestations of such an inhomogeneous phase space structure, can be studied by the method of Peres lattices [46]. The latter are constructed by plotting the expectation 


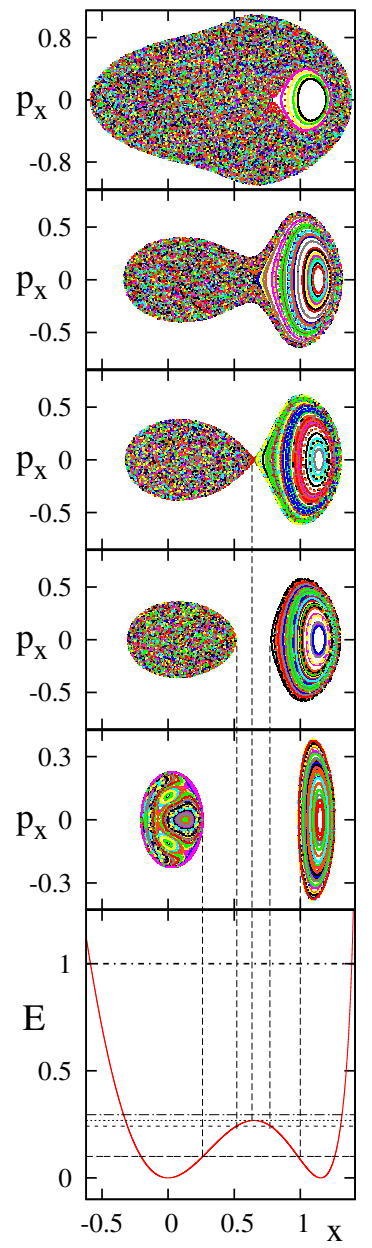

Figure 10. Poincaré sections at the critical point of the first-order QPT (upper five rows). The bottom row displays the classical potential. The five energies at which the sections were calculated consecutively, are indicated by horizontal lines. Adapted from [42].

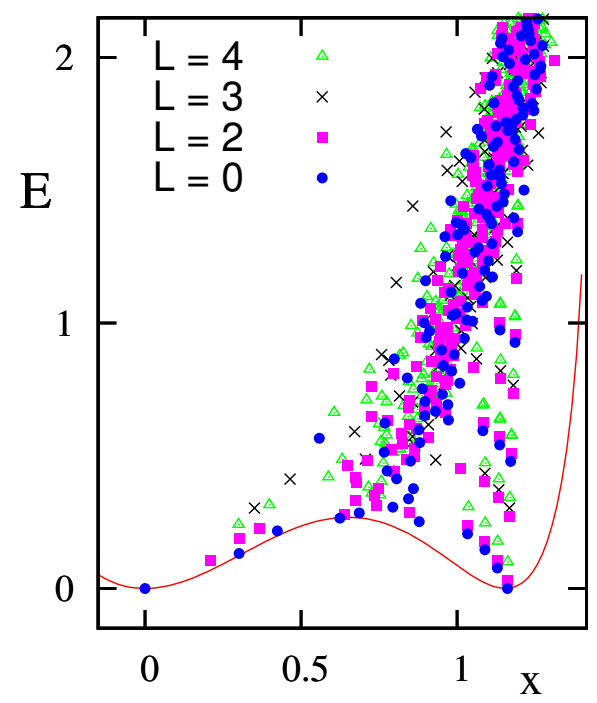

Figure 11. Peres lattices $\left\{x_{i}, E_{i}\right\}$ for $L=0,2,3,4$ and $N=50$ eigenstates of the critical-point Hamiltonian, discussed in the text. The lattices are overlayed on the corresponding classical potential. Adapted from [42].

values $O_{i}=\langle i|\hat{O}| i\rangle$ of an arbitrary operator, $[\hat{O}, \hat{H}] \neq 0$, versus the energy $E_{i}=\langle i|\hat{H}| i\rangle$ of the Hamiltonian eigenstates $|i\rangle$. The lattices $\left\{O_{i}, E_{i}\right\}$ corresponding to regular dynamics display an ordered pattern, while chaotic dynamics leads to disordered meshes of points $[46,47]$. In the present analysis, we choose the Peres operator to be $\hat{n}_{d}$, whose expectation value is related to the coordinate $x$ in the classical potential. The lattices $\left\{x_{i}, E_{i}\right\}$, with $x_{i} \equiv \sqrt{2\left\langle i\left|\hat{n}_{d}\right| i\right\rangle / N}$, can then distinguish regular from irregular states and associate them with a given region in phase space.

The Peres lattices corresponding to $(N=50, L=0,2,3,4)$ eigenstates of $\hat{H}_{\text {cri }}$ are shown in Fig. 11, overlayed on the classical potential. They disclose regular sequences of states localized within and above the deformed well. They are comprised of rotational states with $L=0,2,4, \ldots$ forming regular $K=0$ bands and sequences $L=2,3,4, \ldots$ forming $K=2$ bands. Additional $K$ bands (not shown in Fig. 11), corresponding to multiple $\beta$ and $\gamma$ vibrations about the deformed shape, can also be identified. The states in each regular band share a common structure, to be discussed below. Such ordered band-structures persist to energies above the barrier and are not present in the disordered (chaotic) portions of the Peres lattice. At low-energy, in the vicinity of the spherical well, one can also detect multiplets of states with $L=0, L=2$ and $L=0,2,4$, typical of quadrupole excitations of a spherical shape.

An important clue on the nature of the surviving regular sequences of selected states, in the presence of more complicated type of eigenstates, comes from a symmetry analysis of their wave functions. The left column of Fig. 12 shows the U(5) $n_{d}$-probabilities, $P_{n_{d}}^{\left(L_{i}\right)}$ (13a), for eigenstates of $\hat{H}_{\text {cri }}$, selected on the basis of having the largest components with $n_{d}=0,1,2,3,4$, within the given $L$ spectra. The states are arranged into panels labeled by ' $n_{d}$ ' to conform with 

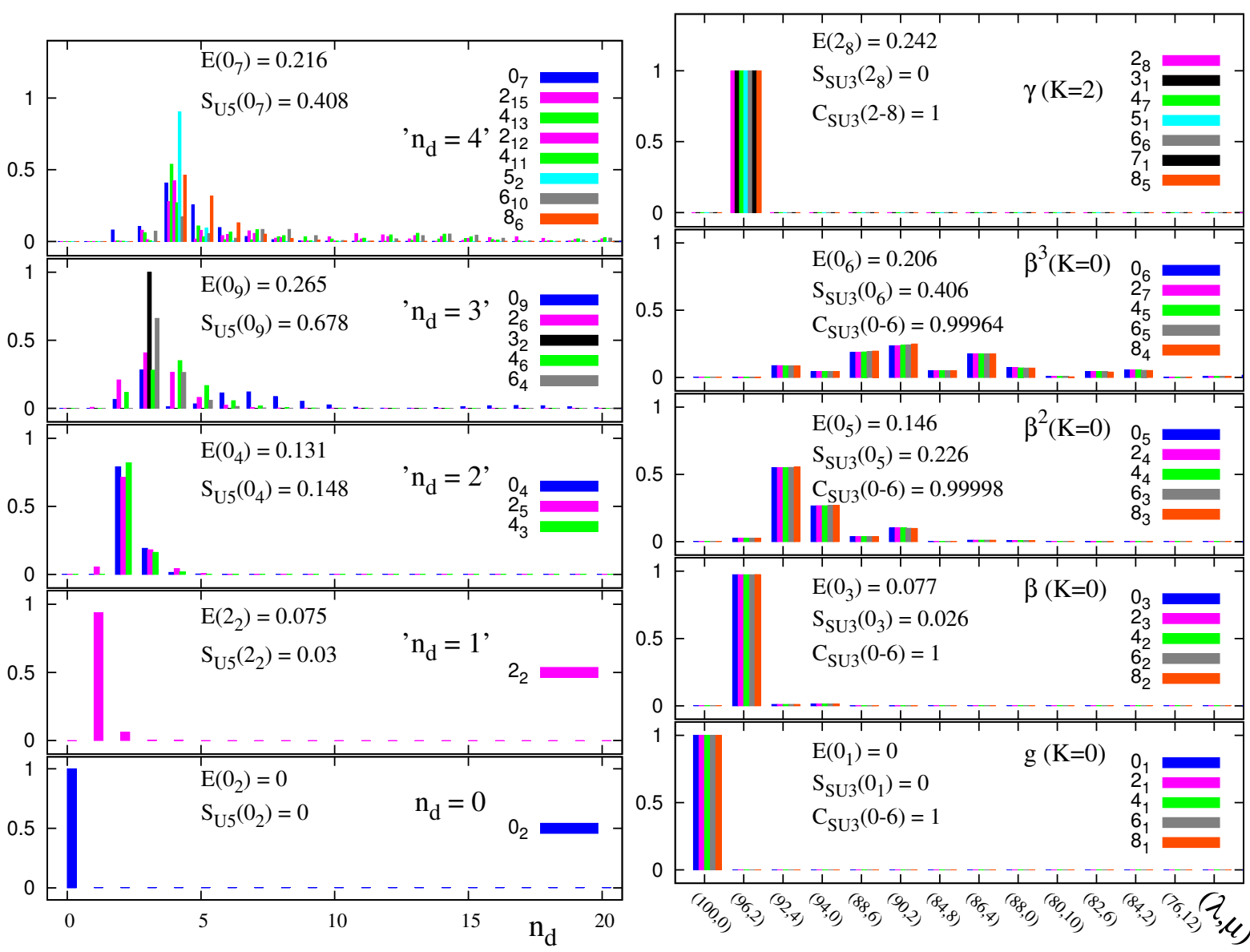

Figure 12. $\mathrm{U}(5) n_{d}$-probability distribution, $P_{n_{d}}^{\left(L_{i}\right)}$ (13a) [left column], and $\mathrm{SU}(3)(\lambda, \mu)$-probability distribution, $P_{(\lambda, \mu)}^{\left(L_{i}\right)}(13 \mathrm{~b})$ [right column], for selected eigenstates of the critical-point Hamiltonian, with $N=50$. The $\mathrm{U}(5)$ Shannon entropy, $S_{\mathrm{U} 5}\left(L_{i}\right)(13 \mathrm{a})$, and $\mathrm{SU}(3)$ correlator, $C_{\mathrm{SU} 3}(0-6)$ are indicated for spherical and deformed type of states, respectively. Adapted from [42].

the structure of the $n_{d}$-multiplets of the U(5) DS limit. The normalized U(5) Shannon entropy $S_{\mathrm{U} 5}\left(L_{i}\right)$, Eq. (13a), is indicated for representative eigenstates. In particular, the zero-energy $L=0_{2}^{+}$state is seen to be a pure $n_{d}=0$ state, with $S_{\mathrm{U} 5}=0$, which is the solvable U(5)-PDS eigenstate of Eq. (16a). The state $2_{2}^{+}$has a pronounced $n_{d}=1$ component $(96 \%)$ and the states $\left(L=0_{4}^{+}, 2_{5}^{+}, 4_{3}^{+}\right)$in the third panel, have a pronounced $n_{d}=2$ component and a low value of $S_{\mathrm{U} 5}<0.15$. All the above states with ' $n_{d} \leq 2^{\prime}$ ' have a dominant single $n_{d}$ component, and hence qualify as 'spherical' type of states. These are the lowest left-most states in the Peres lattices of Fig. 11, mentioned above. In contrast, the states in the panels ' $n_{d}=3$ ' and ' $n_{d}=4$ ' of Fig. 12, are significantly fragmented. A notable exception is the $L=3_{2}^{+}$state, which is the solvable U(5)-PDS state of Eq. (16b) with $n_{d}=3$. The existence in the spectrum of specific spherical-type of states with either $P_{n_{d}}^{(L)}=1\left[S_{\mathrm{U} 5}(L)=0\right]$ or $P_{n_{d}}^{(L)} \approx 1\left[S_{\mathrm{U} 5}(L) \approx 0\right]$, exemplifies the presence of an exact or approximate U(5) PDS at the critical-point.

The states shown on the right column of Fig. 12 have a different character. They belong to the five lowest regular sequences seen in the Peres lattices of Fig. 11, in the region $x \geq 1$. They have a broad $n_{d}$-distribution, hence are qualified as 'deformed'-type of states, forming rotational bands: $g(K=0), \beta(K=0), \beta^{2}(K=0), \beta^{3}(K=0)$ and $\gamma(K=2)$. Each panel depicts the $\operatorname{SU}(3)(\lambda, \mu)$ - 
distribution, $P_{(\lambda, \mu)}^{\left(L_{i}\right)}$ for the band members, the normalized SU(3) Shannon entropy $S_{\mathrm{SU} 3}(L)(13 \mathrm{~b})$ for the bandhead state, and the Pearson correlator $C_{\mathrm{SU} 3}\left(0_{i}-6\right)$ defined in Section 6 . The ground $g(K=0)$ and $\gamma(K=2)$ bands are pure $\left[S_{\mathrm{SU} 3}=0\right]$ with $(\lambda, \mu)=(2 N, 0)$ and $(2 N-4,2)$ SU3 $)$ character, respectively. These are the solvable bands of Eq. (11) with SU(3) PDS. The nonsolvable $K$-bands are mixed with respect to $\mathrm{SU}(3)$ in a coherent, $L$-independent, manner, hence exemplify SU(3)-QDS. As expected, we find $C_{\mathrm{SU} 3}\left(0_{i}-6\right) \approx 1$ for these $K$-bands. The persisting regular $\mathrm{U}(5)$-like [SU(3)-like] multiplets reflect the geometry of the classical Landau potential, as they are associated with the different spherical (deformed) minimum. One can use the corresponding measures of PDS and QDS as fingerprints of the QPT, not only at the critical point, but also throughout the coexistence region, where the two minima interchange [42].

\section{Concluding remarks}

The many examples of PDS and QDS, discussed in the present contribution, demonstrate that these intermediate-symmetries are more abundant than previously recognized. Contrary to naive expectations, the symmetry triangle appears to encompass important elements of symmetry and "not all is lost" inside it. Although, the examples considered were presented in the framework of a bosonic model, it is important to emphasize that these symmetry concepts are applicable to any many-body problem (bosons and fermions) endowed with an algebraic structure. Examples of many-body Hamiltonians with fermionic PDS and QDS are known [48-50]. The PDS algorithms discussed, for constructing Hamiltonians with PDS, are applicable to any semi-simple algebra and can be extended to coupled algebraic structure, $G_{1} \times G_{2}$ [51,52]. Attempts are under way to extend the PDS notion to Bose-Fermi symmetries and supersymmetries [53].

An important virtue of the PDS algorithms is their ability to incorporate and provide a selection criterion for higher-order terms [54]. Such terms are needed for an accurate description of the data and for extensions of ab-initio and beyond-mean-field methods to larger systems, which necessitate a strategy to deal with A-body effective interactions and proliferation of parameters. On one hand, the PDS approach allows more flexibility by relaxing the constrains of an exact dynamical symmetry (DS). On the other hand, the PDS approach picks particular symmetry-breaking terms which do not destroy results previously obtained with a DS for a segment the spectrum. The PDS construction is implemented order by order, yet the scheme is non-perturbative in the sense that the non-solvable states can experience strong symmetrybreaking. These virtues generate an efficient tool which can greatly enhance the scope of algebraic modeling of dynamical systems.

Correlated quantum many-body systems often display an astonishing regular excitation patterns which raises a fundamental question, namely, how simplicity emerges out of complexity in such circumstances. The simple patterns show up amidst a complicated environment of other states. It is natural to associate the "simple" states with a symmetry that protects their purity and special character. This symmetry, however, is shared by only a subset of states, and is broken in the remaining eigenstates of the same Hamiltonian. It thus appears that realistic quantum many-body Hamiltonians can accommodate simultaneously eigenstates with different symmetry character. The symmetry in question cannot be exact but only partial or "apparent". These are precisely the defining ingredients of PDS and QDS. These novel concepts of symmetries can thus offer a possible clue in addressing the "simplicity out of complexity" challenge.

\section{Acknowledgments}

I thank P. Van Isacker, J.E. García Ramos, C. Kremer, N. Pietralla and M. Macek for a fruitful collaboration on the topics discussed and F. Iachello, P. Cejnar and R.F. Casten for insightful discussions. This work is supported by the Israel Science Foundation. 


\section{References}

[1] Bohm A, Néeman Y and Barut A O eds 1988 Dynamical Groups and Spectrum Generating Algebras (Singapore: World Scientific)

[2] Iachello F and Arima A 1987 The Interacting Boson Model (Cambridge: Cambridge Univ. Press)

[3] Iachello F and Levine R D 1994 Algebraic Theory of Molecules (Oxford: Oxford Univ. Press)

[4] Alhassid Y and Leviatan A 1992 J. Phys. A 25 L1265

[5] García-Ramos J E, Leviatan A and Van Isacker P 2009 Phys. Rev. Lett. 102112502

[6] Leviatan A 2011 Prog. Part. Nucl. Phys. 6693

[7] Bahri C and Rowe D J 2000 Nucl. Phys. A 662125

[8] Rowe D J 2004 Nucl. Phys. A 74547

[9] Turner P S and Rowe D J 2005 Nucl. Phys. A 756333

[10] Rosensteel G and Rowe D J 2005 Nucl. Phys. A 75992

[11] Bohr A and Mottelson B R 1975 Nuclear Structure II (Benjamin, Reading, MA)

[12] Ginocchio J N and Kirson M W 1980 Phys. Rev. Lett. 441744

[13] Dieperink A E L, Scholten O and Iachello F 1980 Phys. Rev. Lett. 441747

[14] Warner D D and Casten R F 1983 Phys. Rev. C 28, 1798

[15] Lipas P O, Toivonen P and Warner D D 1985 Phys. Lett. B 155295

[16] Casten R F and Warner D D 1983 Prog. Part. Nucl. Phys. 9311

[17] Leviatan A 1996 Phys. Rev. Lett. 77818

[18] Leviatan A and Sinai I 1999 Phys. Rev. C 60061301

[19] Casten R F, Cakirli R B, Blaum K and Couture A 2014 Phys. Rev. Lett. 113112501

[20] Leviatan A, Novoselsky A and Talmi I 1986 Phys. Lett. B 172144

[21] Cejnar P, Jolie J and Casten R F 2010 Rev. Mod. Phys. 822155

[22] Van Isacker P 1999 Phys. Rev. Lett. 834269

[23] Leviatan A and Van Isacker P 2002 Phys. Rev. Lett. 89222501

[24] Cejnar P and Jolie J 1998 Phys. Lett. B 420 241; ibid 1998 Phys. Rev. E 58387

[25] Kremer C, Beller J, Leviatan A, Pietralla N, Rainovski G, Trippel R and Van Isacker P 2014 Phys. Rev. C $89041302(\mathrm{R})$

[26] McCutchan E A, Zamfir N V and Casten R F 2004 Phys. Rev. C 69064306

[27] Rowe D J , Rochford P and Repka J 1988 J. Math. Phys. (NY) 29572

[28] Rowe D J 2004 in Computational and Group-Theoretical Methods in Nuclear Physics, Escher J, Castaños O, Hirsch J G, Pittel S and Stoitcheva G eds 2004 (Singapore: World Scientific)

[29] Rowe D J 2009 private communication

[30] Van Isacker P 2014 Nuclear Physics News 2423

[31] Macek M, Dobeš J and Cejnar P 2009 Phys. Rev. C 80 014319; ibid 201082014308

[32] Van Isacker P 2014 private communication

[33] Whelan N , Alhassid Y and Leviatan A 1993 Phys. Rev. Lett. 712208

[34] Leviatan A and Whelan N D 1996 Phys. Rev. Lett. 775202

[35] Macek M, Dobeš J, Stránský P and Cejnar P 2010 Phys. Rev. Lett. 105072503

[36] Bonatsos D, McCutchan E A and Casten R 2010 Phys. Rev. Lett. 104022502

[37] Alhassid Y and Whelan N 1991 Phys. Rev. Lett. 67816

[38] Carr L ed. 2010 Understanding Quantum Phase Transitions (CRC press)

[39] Emary C and Brandes T 2003 Phys. Rev. Lett. 90 044101; ibid 2003 Phys. Rev. E 67066203

[40] Macek M and Leviatan A 2011 Phys. Rev. C 84 041302(R)

[41] Leviatan A and Macek M 2012 Phys. Lett. B 714110

[42] Macek M and Leviatan A 2014 Ann. Phys. (NY) 351302

[43] Leviatan A 2006 Phys. Rev. C 74 051301(R)

[44] Leviatan A 2007 Phys. Rev. Lett. 98242502

[45] Hatch R L and Levit S 1982 Phys. Rev. C 25614

[46] Peres A 1984 Phys. Rev. Lett. 531711

[47] Stránský P, Hruška P and Cejnar P 2009 Phys. Rev. E 79066201

[48] Escher J and Leviatan A 2000 Phys. Rev. Lett. 84 1866; ibid 2002 Phys. Rev. C 65054309

[49] Rosensteel G and Rowe D J 2003 Phys. Rev. C 67014303

[50] Van Isacker P and Heinze S 2008 Phys. Rev. Lett. 100 052501; ibid 2014 Ann. Phys. (NY) 34973

[51] Talmi I 1997 Phys. Lett. B 4051

[52] Leviatan A and Ginocchio J N 2000 Phys. Rev. C 61024305

[53] Van Isacker P, Jolie J, Thomas T and Leviatan A 2014 work in progress

[54] Leviatan A, García-Ramos J E and Van Isacker P 2013 Phys. Rev. C 87 021302(R) 